\title{
Estudo da relação estrutura-atividade quantitativa de dibenzoilmetanos $\alpha$-substituídos quanto à atividade anticâncer da mama (linhagem celular MCF7)
}

\author{
A study of quantitative structure-activity relationship in $\alpha$-substituted \\ dibenzoylmethane derivatives as anti-breast cancer agents (MCF7 cell line)
}

\author{
Quirino A. da Lima Neto', Marisa A. Nogueira'2, Ralpho R. dos Reis², Eduardo B. de Melo²
}

\section{Resumo}

O objetivo deste trabalho foi estudar, de forma quantitativa, as relações entre a estrutura química de nove derivados de dibenzoilmetanos e a atividade anticâncer da mama descrita para estes compostos. Foram utilizados 45 descritores físico-químicos. Foi necessário otimizar as estruturas por meio de modelagem molecular, obtendo assim as conformaçôes de menor energia. Após esta etapa, construiu-se uma matriz contendo as variáveis independentes (descritores) e a variável dependente - logaritmo da porcentagem de inibição total (Log \%IT). O próximo passo consistiu em realizar a seleção dos descritores mais correlacionados com Log \%IT para serem utilizados na construção dos modelos. Foram construídos cinco modelos com um descritor e três com dois descritores, pois estes apresentaram R2 > 0,7. A validação externa ficou abaixo do esperado, mas os melhores modelos puderam ser utilizados para a etapa de interpretação físico-química. Verificou-se uma forte influência negativa da hidrofobicidade ( $\log$ P) na atividade biológica. Constatou-se também a importância de propriedades estéricas (RM, RM3 e AR1) e eletrônicas $(\mathrm{Dz})$ para a atividade descrita, também de forma negativa. Porém, a contribuição destas é bem menor, se comparada ao Log P.

Palavras-chave: Neoplasias Mamarias; Linhagem MCF7; Dibenzoilmetanos; Modelagem molecular; Relação estrutura-atividade quantitativa.

\footnotetext{
${ }^{1}$ Ex-graduando, Curso de Farmácia, Universidade Estadual do Oeste do Paraná (UNIOESTE).

${ }^{2}$ Professor, Curso de Farmácia, Universidade Estadual do Oeste do Paraná (UNIOESTE).

Trabalho realizado para obtenção do grau de Farmacêutico por Q.A.L.N.

Enviar correspondência para E.B.M. E-mail: ebmelo@unioeste.br
} 


\section{Abstract}

This study focused on the quantitative structure-activity relationships between nine dibenzoylmethane derivatives and the anti-breast cancer activity described for them. Forty-five physical-chemical descriptors were used. It was necessary to optimize the structures by molecular modeling, thus obtaining lower-energy structures. Following this stage, a matrix was constructed containing the descriptors and the dependent variable (Log \%IT). The next step was to select the descriptors most closely correlated with Log \%IT to be used in constructing the models. Five models with one descriptor and three with two descriptors were constructed, since they presented R2 $>0,7$. External validation was lower than expected, but the best models were used for the physical-chemical interpretation phase. A strong and negative influence of hydrophobicity $(\log P)$ on biological activity was observed. The importance of steric (RM, RM3, and AR1) and electronic (Dz) properties was evident for the described activity, also in negative form. However, their impact was small as compared to Log P.

Key words: Breast cancer; MCF7 cell lines; Dibenzoylmethanes; Molecular modeling; Structure-activity relationships.

\section{INTRODUÇÃO}

O câncer é caracterizado pela multiplicação descontrolada e propagação de formas anormais de células do próprio corpo do indivíduo. Trata-se de uma das principais causas de morte nos países desenvolvidos. Segundo estimativas, uma em cada cinco mortes na Europa e E.U.A. é causada por um dos diversos tipos de câncer. ${ }^{1}$

Em virtude de ser uma das mais importantes causas de morte em mulheres, o câncer da mama é uma das neoplasias mais extensivamente estudadas. Ocorre em ambos os sexos, mas com uma prevalência 100 vezes maior nas mulheres. ${ }^{2}$ No Brasil, é o câncer que mais causa mortes entre as mulheres. Em 2002, ocorreram 10,15 mortes por 100.000 mulheres. Em 2003, foram registrados 41.610 novos casos e 9.335 óbitos. De acordo com as Estimativas de Incidência de Câncer no Brasil para 2006, o câncer da mama será o segundo mais incidente, com 48.930 novos casos. ${ }^{3,4}$

Atualmente, além da cirurgia e da quimioterapia, ${ }^{5,6}$ estão disponíveis alguns fármacos para a quimioterapia do câncer da mama: mostardas nitrogenadas, ciclofosfamida, ifosfamida, tiotepa, metotrexato, fluouracil, vimblastina, taxol, docetaxel, etoposida, teniposida, caproato de hidroxiprogesterona, dietilestilbestrol, propionato de testosterona e tamoxifeno compõem este arsenal terapêutico. ${ }^{5-8}$

Dentre estes, o tamoxifeno (\{2-[4-(1,2-difenil-but-1-enil)fenóxi]-etil\}-dimetil-amina, TMX, figura 1) é o mais amplamente utilizado na prática clínica. Vários estudos comprovam seus efeitos benéficos. Pertence ao grupo dos compostos trifeniletilênicos e é derivado do mesmo núcleo estilbeno que o dietilestilbestrol. Os compostos desse grupo possuem várias atividades estrogênicas e antiestrogênicas. ${ }^{9}$

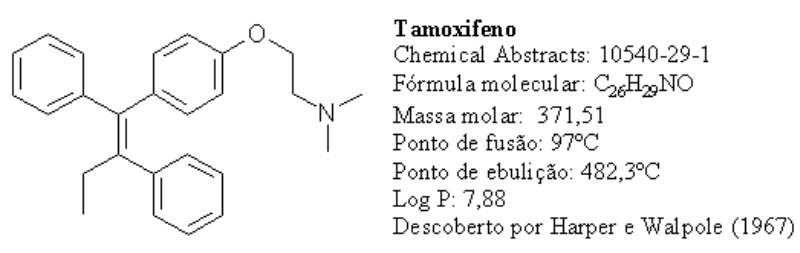

Figura 1. Estrutura e informações básicas do tamoxifeno $(T M X)^{10,11}$

A cada dia, novos compostos naturais ou sintéticos farmacologicamente ativos contra os diversos tipos de tumores são descritos. Mesmo que a grande maioria não se torne um fármaco (composto capaz de ser utilizado na prática clínica), praticamente todos podem ser considerados protótipos de novas entidades moleculares (N.E.M) terapeuticamente úteis. Sendo assim, utilizando estes compostos como ponto de partida, e por meio de modificaçōes moleculares em suas estruturas químicas, podese chegar a novos agentes antitumorais passíveis de utilização na quimioterapia ou quimioprevenção do câncer. ${ }^{12,13}$

Entre as diversas classes de compostos descritas na literatura que apresentam atividade antitumoral, in vitro $\mathrm{e}$ in vivo, encontram-se os dibenzoilmetanos. O dibenzoilmetano (1,3-difenil-propano-1,3-diona, DBM, figura 2) é uma $\beta$-dicetona muito estudada. ${ }^{14-18}$ Este composto e seus derivados formam um grupo raro de pequenas $\beta$-dicetonas que apresentam diversas propriedades farmacológicas, como fotoproteção, atividades antiinflamatória, antitumoral, antiviral e outras. Estão presentes em um grande número de famílias de plantas (Annonaceas, Astraceas, Menispermaceae, Rosaceae, Salicaceae e, especialmente, Leguminoseae), ${ }^{19}$ principalmente carregando isoprenóides e furano ligado aos anéis aromáticos, e, mais raramente, um radical alil 
ou outro ligado ao carbono central da cadeia alifática. ${ }^{20}$ $\mathrm{Na}$ natureza, os representantes dos dibenzoilmetanos são a curcumina [1,7-bis-(4-hidroxi-3-metóxi-fenil)hepta-1,6-dieno-3,5-diona, CCM, figura 2], o pongamol [1-(4-metóxi-benzofuran-5-il)-3-fenil-propano-1,3-diona, PGM, figura 2] e seus derivados. A CCM foi isolada das raízes da Curcumina longa, que é utilizada como tempero na cozinha oriental (curry). O PGM foi a primiera $\beta$-dicetona de ocorrência natural descrita, e foi isolada da casca da raiz da Tephrosia lenceolata, uma planta indiana. ${ }^{21}$ Este composto foi descrito em diversas outras espécies, como a Pongamia glabra, ${ }^{22}$ T. purpurea, T. hamiltoni, ${ }^{23}$ Dablstedtia pinnata, D. pentaphyla ${ }^{24} \mathrm{e}$ Millettia peguensis Ali. $^{25}$

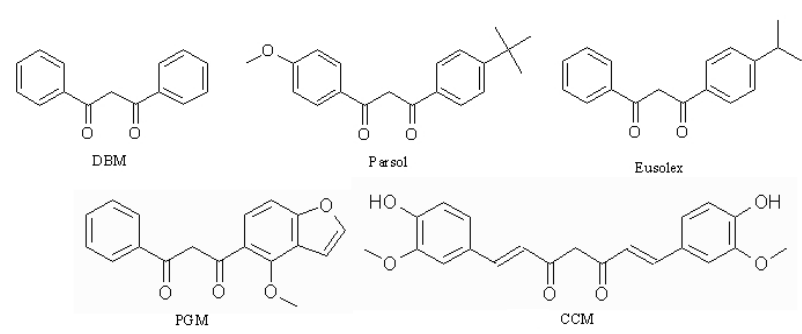

Figura 2. Estruturas do DBM, PGM, CCM, Parsol e Eusolex

Dois derivados de DBM são utilizados comumente na indústria cosmética devido a suas propriedades fotoprotetoras: o Eusolex ${ }^{\circledR}$ [1-(4-isopropil-fenil)-3-fenilpropano-1,3-diona] e o Parsol® [1-(4-tert-butil-fenil)-3(4-metóxi-fenil)-propano-1,3-diona] (figura 2). ${ }^{26}$

A ciência envolvida com o desenho, com a descoberta e o desenvolvimento de novos fármacos é a química ou medicinal ou farmacêutica. Nesta área, os estudos de relações estrutura-atividade (SAR: Structure-Activity Relationships) são utilizados para definir os parâmetros importantes para a ação de uma classe de compostos análogos contra certa patologia, pois parte da premissa geral de que uma droga age em um sítio de ação específico localizado em uma enzima ou receptor, sendo que compostos com estruturas semelhantes tendem então a possuir a mesma atividade farmacológica e/ou toxicológica, ou seja, interagem nesse mesmo sítio. ${ }^{27}$

O sucesso da abordagem SAR no desenho de fármacos depende não somente do conhecimento e da experiência da equipe de desenho, mas também de uma boa dose de sorte. O estudo de relações estruturaatividade quantitativas (QSAR: Quantitative StructureActivity Relationships) procura remover o elemento sorte ao estabelecer uma relação matemática, sob a forma de uma equação, entre a atividade biológica e os parâmetros (ou propriedades) físico-químicos mensuráveis que possuam influência sobre a atividade terapêutica de uma classe de compostos. ${ }^{27}$

O estudo de QSAR, como é conhecido hoje, propõe que a ação de uma droga pode ser dividida em dois estágios: o transporte para seu sítio de ação e a ligação ao mesmo. Assim, a atividade biológica está associada às mudanças de energia livre que ocorrem nos processos de absorção, distribuição e biotransformação ou à própria interação droga-receptor, sendo esse o paradigma que fundamenta o estudo de QSAR. ${ }^{27}$ Este fenômeno poderia ser explicado por modelos matemáticos simples ou multiparamétricos, em que a atividade biológica pode ser determinada por parâmetros que medem a influência de cada uma das propriedades, como demonstrado abaixo na equação 1 :

$$
A B=\alpha \sigma+\beta \pi+c E s+d
$$

$\mathrm{AB}$ é a atividade biológica; $\sigma, \pi$ e $E s$ são constantes de substituintes referentes à distribuição eletrônica e à hidrofobicidade (razão entre o quanto a molécula é solúvel em meio apolar e meio polar, o que influencia diretamente na absorção e distribuição da molécula no organismo), bem como às características estéricas (propriedades relacionadas à forma e tamanho da molécula), respectivamente; a, b, c expressam a contribuição de cada propriedade para a atividade biológica; d é uma constante. ${ }^{27}$ Cada uma dessas propriedades contribui com maior ou menor intensidade para o estabelecimento da resposta biológica e pode ser representada por descritores estruturais que expressam a sua influência na bioatividade de compostos químicos. ${ }^{28-31}$

Atualmente, o desenvolvimento de uma nova droga ou fármaco, clinicamente útil, pode chegar a 15 anos, devido aos custos e tempo necessários. Os estudos de QSAR são uma das mais importantes contribuições na área de química medicinal, visto que eles visam à identificação de compostos protótipos e dão embasamento físico-químico para o planejamento de análogos mais específicos, com maior atividade intrínseca ou com melhor perfil farmacológico, aumentando, desta forma, as chances de sucesso de inserção de novos fármacos no arsenal terapêutico. ${ }^{27}$ Estes estudos são, mais comumente, realizados utilizando programas de computadores que calculam as propriedades físico-químicas mensuráveis de substâncias químicas (quando os cálculos são realizados com as estruturas tridimensionais dos compostos que estão sendo estudados, tem-se o processo denominado modelagem molecular ${ }^{29}$ ) e que avaliam a relação destas com a atividade biológica, gerando equações de regressão ou modelos de previsão. Estes estudos integram, dentro da química medicinal e junto a outras abordagens, a área conhecida como planejamento de fármacos auxiliado por 
computador (CADD: Computer Aided Drug-Design). ${ }^{28,29}$

Considerando o exposto, este trabalho teve como objetivo realizar um estudo QSAR baseado em nove dibenzoilmetanos $\alpha$-substituídos (figura 3) sintetizados e testados por Nogueira et al..$^{32}$ quanto à inibição da linhagem celular de tumores da mama MCF7. Assim, através dos parâmetros físico-químicos mais importantes para o efeito descrito, poderá ser obtida uma equação (ou equaçôes) que auxilie $(\mathrm{m})$ no planejamento $\mathrm{da}(\mathrm{s})$ síntese(s) de novos derivados.

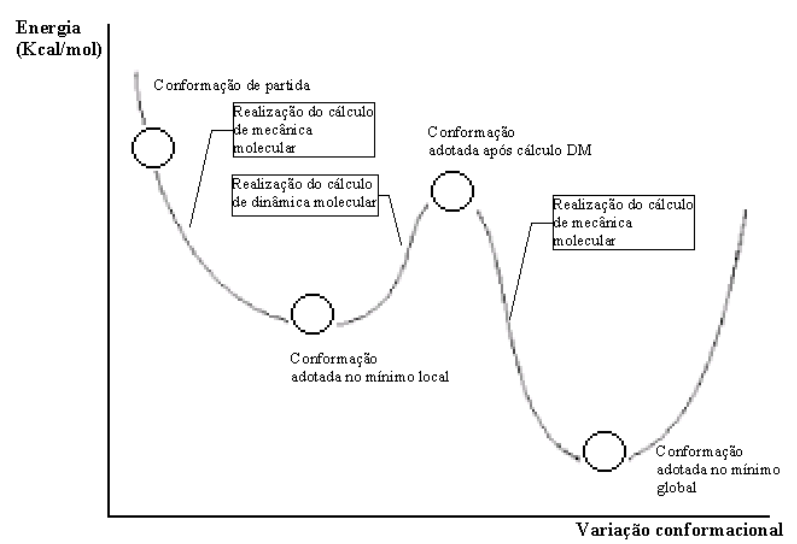

Figura 3. Protótipos utilizados para o presente estudo. Compostos 1 a 9 foram utilizados para a montagem dos modelos QSAR, enquanto 10 a 12 para a validação dos modelos

\section{MATERIAL E MÉTODOS}

As seguintes etapas foram realizadas: (I) modelagem molecular dos compostos pertencentes ao grupo de estudo (1-9) e do grupo a ser utilizado para o processo conhecido como validação externa (10-12); ${ }^{33}$ (II) obtenção dos descritores físico-químicos; e (III) montagem e validação dos modelos de previsão.

Para as etapas I e II, foi utilizado o programa de modelagem molecular HyperChem 7.1.34 Para a etapa III, o programa de análise de Hansch BuildQSAR ${ }^{35}$ e a planilha de cálculos do OppenOffice 1.1.5. ${ }^{36}$

Uma explanação da forma e a importância destes procedimentos serão apresentadas nos próximos itens, mas incentivamos os interessados em maiores informaçōes sobre este tema a consultar diversos artigos publicados na revista Química Nova (www.scielo.br/qn). 28-30, 37-42

\section{MOdeLAGEM MOLECULAR}

Os dibenzoilmetanos, quando apresentam hidrogênio em R3 (carbono $\alpha$ ), existem exclusivamente na forma de um tautômero ceto-enólico, apresentando um deslocamento do equilíbrio para a forma enólica graças à formação de uma ponte de hidrogênio intramolecular. ${ }^{43,44}$ Isto não ocorre nos compostos aqui estudados, devido a uma tensão estérica entre o substituinte em $\mathrm{R} 3$ e os radicais fenila, que se encontrariam no mesmo plano quando na forma ceto-enólica. Assim, o equilíbrio favorece o tautômero $\beta$-dicetona (figura 4). ${ }^{45}$ Considerando estes dados, esta forma foi a escolhida para o processo de modelagem molecular dos compostos 1 a 9 .

Como o ensaio biológico ${ }^{32}$ foi realizado com a mistura racêmica dos compostos 4 a 12 , a configuração tridimensional absoluta utilizada, de forma padrão, foi a (R) para todos os carbonos assimétricos.

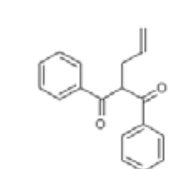

(1)

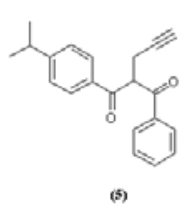

(5)

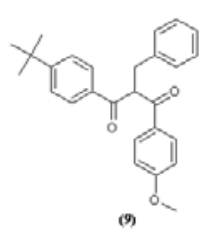

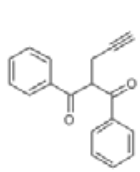

(2)

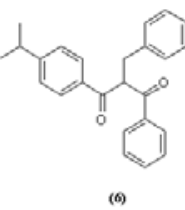

(6)

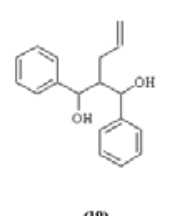

(10)

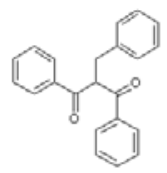

(3)

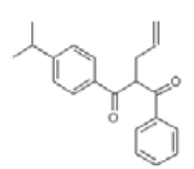

(4)

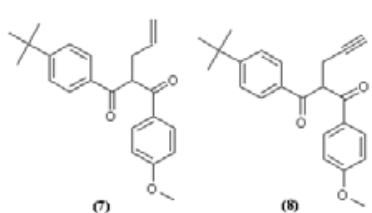

()

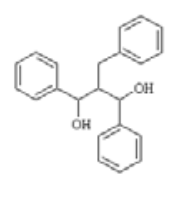

(11)

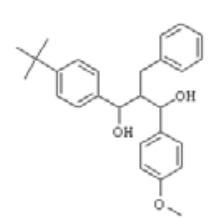

(12)
Figura 4. Equilíbrio tautomérico dos dibenzoilmetanos. Se R3 = $\mathrm{H}$, o equilíbrio é deslocado para direita. Se for diferente, é deslocado para a esquerda

Os 12 compostos estudados tiveram suas conformações tridimensionais termodinamicamente mais estáveis calculadas no programa HyperChem 7.1..$^{34}$

Em química medicinal, considera-se que dentre as conformaçōes mais estáveis (ou de menores energias) da molécula isolada deve estar a bioativa (aquela que se liga ao sítio de ação), assim como as conformações da mesma quando em solução e em sua forma cristalina. Porém, não será necessariamente a mais estável. Assim, quando não há algum dado experimental que indique qual é realmente a conformação bioativa, é utilizada, de forma padrão, a mais estável obtida pelo processo conhecido como otimização da geometria por modelagem molecular. ${ }^{46}$

Os descritores que não são baseados na estrutura tridimensional dos compostos são obtidos por simples contagem ou por cálculos convencionais. Caso contrário, há a exigência de que se faça a otimização da geometria de cada um dos compostos, pois, como a conformação mais estável é aquela considerada bioativa, as propriedades desta serão as importantes para o estudo de QSAR. ${ }^{46}$

Após o desenho de cada molécula, foram realizados 
cálculos de mecânica molecular (MM), base matemática que trata as moléculas como uma coleção de átomos unidos por forças harmônicas ou elásticas (forças newtonianas). Essas forças podem ser descritas como funções de energia potencial de características estruturais, como comprimentos de ligação, ângulos de ligação, interaçôes não-ligantes e outras. Devido a isto, estes são conhecidos como cálculos de campo de força. A rapidez, a economia de tempo de computação e a facilidade de compreensão são algumas das vantagens da mecânica molecular. ${ }^{47}$

No programa HyperChem 7.1, foi usado o campo de força $\mathrm{MM}_{+}$, de N. L. Allinger. Nesse, a partir de um conjunto de dados de entrada, uma geometria inicial é especificada e sua energia é calculada. Todos os parâmetros que definem a geometria do sistema são modificados em incrementos pequenos e, pelo uso de métodos de abaixamento de gradiente, a geometria é otimizada. No entanto, esse método de otimização depende da geometria de partida, o que faz com que a minimização geralmente leve ao mínimo local (uma conformação estável, porém não a de menor energia) mais próximo e não ao mínimo global (a conformação mais estável entre todas aquelas que uma molécula pode adotar) (figura 5). ${ }^{47}$

Um método capaz de ultrapassar pequenas barreiras, portanto, mais eficiente do que a otimização simples na localização de um mínimo local mais profundo, são os cálculos de dinâmica molecular (DM). Eles simulam o movimento baseado em cálculos de energia potencial, utilizando o campo de força e as equações de Newton para movimento, considerando cada átomo uma partícula, assim como na mecânica molecular. O método consiste em simular a colocação da molécula em um banho de temperatura e viscosidade definidas. É dado um intervalo de tempo em que ocorre a transferência de calor entre o banho e a molécula e outro intervalo de tempo em que a molécula equilibra a sua energia. $\mathrm{O}$ processo é repetido o número de vezes especificado pelo usuário. ${ }^{47}$ A partir do resultado da DM, onde é obtida uma conformação de energia um pouco maior do que o mínimo local, pode-se chegar, por repetição da $\mathrm{MM}$, a um mínimo local de energia ainda menor, ou, finalmente, ao mínimo global (figura 5).

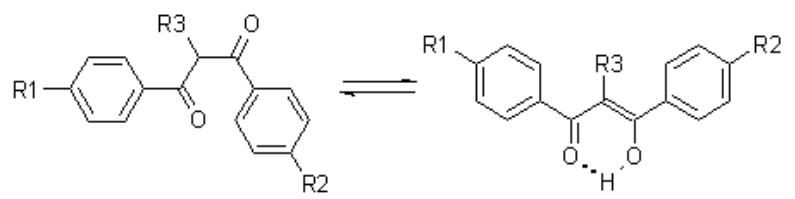

Figura 5. Representação da superfície de energia potencial obtida na otimização da geometria de uma molécula. As circunferências representam as conformações adotadas em cada etapa. Esquema baseado na referência 41
Os cálculos de dinâmica molecular foram simulados em um banho de $300 \mathrm{~K}$, cuja transferência de calor se deu durante 1 ps (picosegundo). Assim, os cálculos MM e DM foram realizados alternadamente, conforme o esquema apresentado na figura 5 , até que se obtivesse a conformação de menor energia.

No entanto, apesar da MM ser uma boa técnica de otimização da geometria para moléculas pequenas, ela não oferece informações quanto às características eletrônicas dos compostos. Portanto, há a necessidade de se usar cálculos de mecânica quântica para um estudo mais completo das estruturas. ${ }^{48}$ Uma molécula cuja geometria foi otimizada por MM pode ser utilizada como ponto de partida para cálculos de orbitais moleculares por meio de mecânica quântica, ${ }^{47}$ como foi realizado neste estudo.

Por muito tempo, os físicos acreditaram que a mecânica clássica (a base dos cálculos $\mathrm{MM}$ ) não descrevia corretamente o comportamento de partículas muito pequenas, como os elétrons e os núcleos dos átomos e moléculas. Assim, diversos pesquisadores começaram a desenvolver uma nova área do conhecimento denominada posteriormente de mecânica quântica. ${ }^{47}$

Para descrever o estado de um sistema, em mecânica quântica, foi postulada a existência de uma função de coordenadas, chamada função de onda $(\psi)$. A função de onda contém todas as informaçóes sobre a molécula, e a partir dela podem ser calculadas todas as suas propriedades eletrônicas. ${ }^{47}$

Os cálculos de mecânica quântica não são exatos, pois são feitas algumas aproximaçôes baseadas em dados empíricos, e podem ser subdivididos em dois métodos: ab initio e semi-empírico. O primeiro pode ser aplicado apenas a moléculas pequenas e, apesar de mais preciso e não precisar de dados armazenados, requer grande capacidade de memória e tempo de cálculo do computador. ${ }^{41,48} \mathrm{O}$ segundo, uma alternativa ao ab initio, é mais simplificado e apresenta valores aproximados que vêm armazenados no programa, sendo estes dois fatores determinantes na redução do tempo gasto no cálculo computacional. ${ }^{48}$

Um grande número de métodos semi-empíricos com aplicaçôes diversas foi desenvolvido nas últimas duas décadas. Para as estruturas em estudo, o método selecionado foi o AM1 (Austin Model 1). Uma das vantagens desse método é a disponibilidade de parâmetros para uma grande variedade de átomos e resultados mais exatos para cálculos de carga, momento de dipolo e energia de orbitais moleculares, ${ }^{49-52}$ descritores utilizados neste estudo. A metodologia aqui adotada foi a mesma utilizada nos cálculos por MM, ou seja, otimização por AM1 alternado com DM, até a 
estabilização da menor energia. A geometria obtida no final da etapa foi considerada a conformação mais

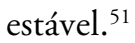

\section{OBTENÇÃO DOS DESCRITORES FÍSICO-QUÍMICOS}

A partir das geometrias otimizadas pelo método AM1 implementado no programa HyperChem 7.1, foram obtidas 40 variáveis, ou descritores físico-químicos, dos compostos 1 a 9 (tabela 1). Estas variáveis foram obtidas com a utilização do módulo QSAR Properties, que fornece uma série de descritores empíricos (eletrônicos, estéricos e hidrofóbicos) baseados em estudos de diversos autores, ${ }^{53}$ e pelas opções Properties e Orbitlals do menu Compute, e pela opção Charges, do menu Display $\rightarrow$ Labels, que forneceram as variáveis de distribuição eletrônica calculadas por AM1.

\section{MONTAGEM DOS MODELOS DE PREVISÃO}

Para a montagem dos modelos de previsão quantitativos, é necessária uma variável dependente experimental. Neste caso, o logaritmo da porcentagem de inibição total (Log \% IT) in vitro da linhagem celular MCF7 foi determinado a partir dos estudos experimentais realizados por Nogueira et al..$^{32} \mathrm{~A} \% \mathrm{IT}$ foi obtida a partir da soma das porcentagens de inibição do crescimento e da citotoxicidade dos compostos testados (\%IT = \% IC + \%CC, tabela 2$)$, e fornece uma idéia da potência total de cada composto.

A seleção das melhores combinações de variáveis ${ }^{42}$ foi realizada utilizando algoritmo genético ${ }^{42}$ implementado no BuildQSAR.

Com a determinação das combinaçôes de variáveis mais promissoras, construíram-se modelos, os quais foram avaliados para verificar se a sua especificação adapta-se convenientemente aos dados observados. Essa avaliação foi dividida em três partes: avaliação do grau de ajuste, do grau de significância e do grau de previsibilidade. ${ }^{38}$

O primeiro é medido em termos de sua capacidade de reproduzir o valor observado dos objetos. A avaliação do grau de ajuste é feita através do cálculo do coeficiente de correlação R e do desvio-padrão $s$ para cada modelo, além da análise dos resíduos, calculado a partir da diferença do Log \%IT observado experimentalmente $\left(\log \% \mathrm{IT}_{\mathrm{Obs}}\right)$ e do Log \%IT previsto pelo modelo (Log $\left.\% \mathrm{IT}_{\text {Prev }}\right)$. O que se espera de um modelo em relação ao grau de ajuste é que ele apresente $\mathrm{R}$ o mais próximo possível de 1 (ou -1, no caso de uma regressão inversa), que o valor de $s$ seja o mais próximo possível de zero e que os resíduos apresentem distribuição normal em torno de zero. Também relacionado ao grau de ajuste do modelo, tem-se o quadrado do coeficiente de correlação $\mathrm{R} 2$, o qual corresponde à fração da variabilidade total que é explicada pelo modelo. Por exemplo, um modelo de QSAR em que $R^{2}=0,9$ é dito capaz de explicar $90 \%$ da variabilidade total dos valores observados da atividade biológica, em torno de sua média. ${ }^{38}$

Determinou-se que seriam avaliados os graus de significância e previsibilidade apenas dos modelos que apresentassem um valor de $R^{2}$ acima de 0,7 para correlação com Log \% $\mathrm{IT}_{\mathrm{Obs}}$ e, no caso daqueles que apresentavam duas variáveis, um valor de $R^{2}$ menor do que 0,7 para a correlação entre as variáveis presentes no modelo, pois, do contrário, estaria se perdendo tempo com modelos inapropriados para o estudo. ${ }^{42} \mathrm{~A}$ literatura ${ }^{37-}$ ${ }^{42}$ geralmente recomenda $\mathrm{R}>0,7$ (ou seja, $\mathrm{R} 2>0,5$ ) como o limite mínimo aceitável, mas este grupo optou por aumentar os critérios de exigência, aplicando este valor numérico a $R^{2}$, visando a chegar a modelos com alta capacidade preditiva e alto nível de confiança.

O grau de significância é medido através da execução de testes de validação (teste estatístico de hipótese). Para testar a significância estatística do $R^{2}$, aplica-se um teste de hipótese conhecido como teste $F$. Esse verifica o quanto da variabilidade da atividade observada experimentalmente, $\log \% \mathrm{IT}_{\mathrm{Obs}}$, pode ser explicada pelas variáveis $X 1, X 2, \ldots, X k$. Para validar $R^{2}$ através do teste $F$, é preciso comparar o valor de $F$ obtido no modelo com o valor de referência. Esse, em geral, se refere ao nível de confiança de $95 \%$ e pode ser obtido em tabelas apropriadas. ${ }^{38,56}$

O grau de previsibilidade do modelo é testado através da validação cruzada. Um modelo, com elevado grau de previsibilidade, para objetos não incluídos no mesmo, apresentará $Q^{2}$ (quadrado do coeficiente de correlação da validação cruzada) próximo de 1 e SPRESS (desvio padrão da validação cruzada) próximo de zero. ${ }^{38}$ Muitos autores consideram valores acima de 0,5 para o $Q^{2}$ como um bom indicador, ou mesmo a prova final de que o modelo é altamente preditivo. ${ }^{33}$ As equaçóes utilizadas para o cálculo destes parâmetros são apresentadas na figura 6 .

A simples obtenção de um modelo QSAR pode significar muito pouco no que diz respeito à sua validade e significado. Mesmo após a obtenção de bons resultados nas etapas anteriores, é necessária a realização da validação externa para introduzir maior confiança no modelo. ${ }^{37} \mathrm{~A}$ validação externa (VE) foi feita neste trabalho com os compostos 10, 11 e 12 (figura 3), que também foram sintetizados e testados por Nogueira et al. ${ }^{32}$ A mesma metodologia de modelagem molecular aplicada a 1-9 foi utilizada, obtendo assim os parâmetros necessários à resolução dos modelos propostos, chegando por fim a valores de $\log \% \mathrm{IT}_{\mathrm{VE}}$ para os compostos 10 , 11 e 12. Essas atividades previstas foram então correlacionadas com os valores de $\mathrm{Log} \% \mathrm{IT}_{\mathrm{Obs}}$, obtendo- 
Tabela 1. Classificação e descrição das variáveis utilizadas no estudo

\begin{tabular}{|c|c|c|}
\hline DESCRITORES & CLASSE & FONTE \\
\hline Logaritmo do coeficiente de partição óleo/água (Log P) & Hidrofóbico & QSAR Properties \\
\hline Refratividade molar (RM) & Misto (estérico/eletrônico) & QSAR Properties \\
\hline Refratividade molar do substituinte R 1 & Misto (estérico/eletrônico) & QSAR Properties \\
\hline Refratividade molar do substituinte R2 & Misto (estérico/eletrônico) & QSAR Properties \\
\hline Refratividade molar do substituinte R3 & Misto (estérico/eletrônico) & QSAR Properties \\
\hline Massa molar (MM) & Estérico & QSAR Properties \\
\hline Volume molecular total (VM) & Estérico & QSAR Properties \\
\hline Volume do substituinte $R$ & Estérico & QSAR Properties \\
\hline Volume do substituinte R2 & Estérico & QSAR Properties \\
\hline Área total $(A)$ & Estérico & QSAR Properties \\
\hline Área do substituinte R1 & Estérico & QSAR Properties \\
\hline Área do substituinte R2 & Estérico & QSAR Properties \\
\hline Área do substituinte R3 & Estérico & QSAR Properties \\
\hline Momento dipolar (D) & Eletrônico & AM1 \\
\hline Momento dipolar nos eixos $x(1)$ & Eletrônico & AM1 \\
\hline Momento dipolar nos eixos y (2) & Eletrônico & AM1 \\
\hline Momento dipolar nos eixos z (3) & Eletrônico & AM1 \\
\hline $\begin{array}{l}\text { Energia do orbital molecular mais alto } \\
\text { ocupado (HOMO) }\end{array}$ & Eletrônico & AM1 \\
\hline $\begin{array}{l}\text { Energia do orbital molecular mais } \\
\text { baixo desocupado (LUMO) }\end{array}$ & Eletrônico & AM1 \\
\hline $\begin{array}{l}\text { Diferença das energias entre os orbitais } \\
\text { LUMO e HOMO (Gap) }\end{array}$ & Eletrônico & $\mathrm{AM} 1$ \\
\hline $\begin{array}{l}\text { Cargas parciais }\left(Q_{N}\right) \text { dos átomos } \\
1 \text { a } 18\left(Q_{1} a Q_{18}\right)\end{array}$ & Eletrônicos & AM1 \\
\hline $\begin{array}{l}\text { Somatório das cargas parciais dos } \\
\text { substituintes } R 1 \text {, R2 e R3 }\left(\sum_{R 1, R 2 \text { e R3 }}\right)\end{array}$ & Eletrônico & AM1 \\
\hline Calor de formação (CF) & Termodinâmico & AM1 \\
\hline
\end{tabular}

se o quadrado do coeficiente de correlação para a validação externa $\left(R_{V E}^{2}\right)$. Da mesma forma que o $R^{2}$, determinou-se que o $R_{V E}^{2}$ deveria apresentar valor mínimo de 0,7 , o que garantiria modelos com alta capacidade de predição para novos derivados. A correlação de $\log \% \mathrm{IT}_{\text {Prev }}$ foi realizada na planilha de cálculos do programa OppenOffice 1.1.5. ${ }^{36}$

\section{RESULTADOS E DISCUSSÃO}

Segundo a metodologia adotada, 8 modelos foram selecionados para a avaliação, 5 formados por um descritor e 3 formados por dois descritores.

A equação 2 apresenta o melhor modelo obtido com um descritor. 
Tabela 2. Valores para porcentagem de inibição do crescimento celular $(\% \mid C)$ e de citotoxicidade $(\% \mathrm{CC})$ e somatório desses valores $(\% \mid T)$ quanto à linhagem celular

\begin{tabular}{ccccc}
\hline COMPOSTO & $\%$ IC & $\%$ CC & $\%$ LT & Log IT \\
\hline 1 & 100,0 & 30,0 & 130,0 & 2,114 \\
2 & 100,0 & 10,0 & 110,0 & 2,041 \\
3 & 83,3 & 0,0 & 83,3 & 1,921 \\
4 & 100,0 & 60,0 & 160,0 & 2,204 \\
5 & 100,0 & 5,0 & 105,0 & 2,021 \\
6 & 100,0 & 33,3 & 133,3 & 2,125 \\
7 & 83,3 & 0,0 & 83,3 & 1,921 \\
8 & 60,0 & 0,0 & 60,0 & 1,778 \\
9 & 40,0 & 0,0 & 40,0 & 1,600 \\
\hline
\end{tabular}

'Ensaios realizados conforme metodologia descrita por Alley et al. ${ }^{41}$

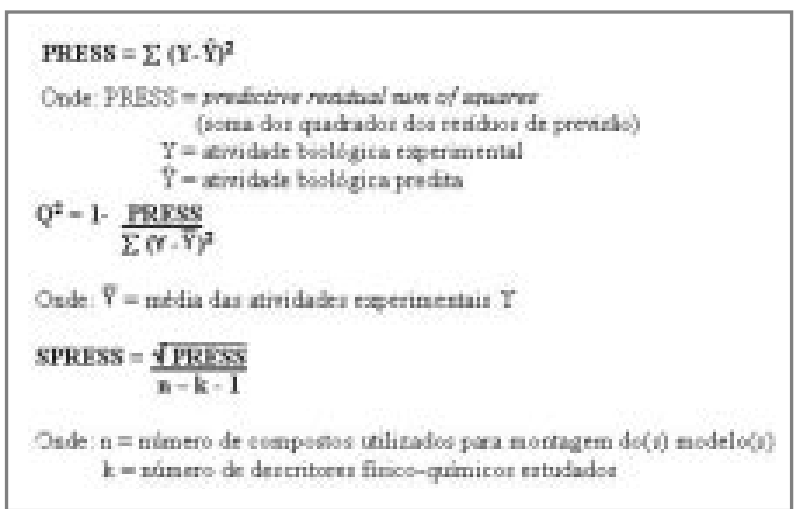

Figura 6. Equações utilizadas para resolução do Q 2 e SPRESS. Figura baseada em dados apresentados na referência 38

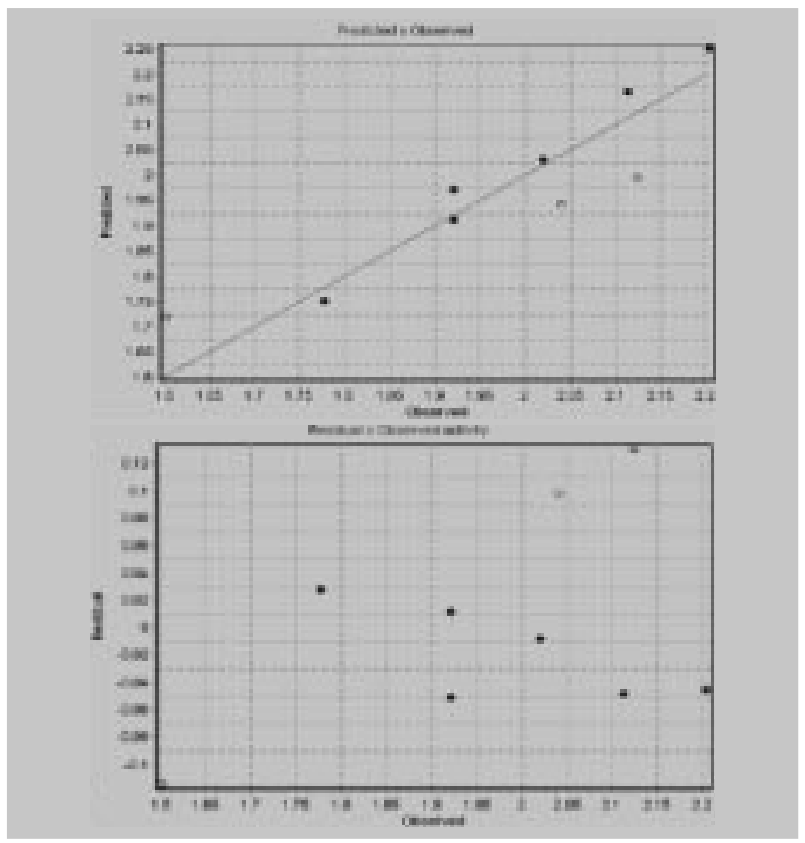

Figura 7. $L$ og $\% \mathrm{IT}_{\mathrm{obs}}$ em função de $\mathrm{Log} \% \mathrm{IT}$ Prev e resíduos de regressão em função de Log \%IT para a equação 2 $\log \%$ IT $=-0,18496( \pm 0,07267)$ LogP $+2,88029( \pm 0,363420)$ $n=9 ; R^{2}=0,831 ; s=0,083 ; F=34,444 ; Q 2=0,701 ;$ SPRESS $=0,110$

0 modelo de regressão representado pela equação 2 é capaz de explicar cerca de $83 \%$ da variabilidade observada na atividade $\left(R^{2} \times 100\right)$. Gaudio e Zandonade ${ }^{38}$ consideram este nível de ajuste excelente, fato esse confirmado pelo baixo valor do desvio-padrão s. Esses valores podem ser objetivamente analisados em termos de dois gráficos: Log \%IT obs $_{\text {en função de } L o g} \% I T_{\text {Prev }}$ e resíduos de regressão em função de $\log \% I T_{\text {obs }}$ (figura 7).

No gráfico do Log \%IT obs' em função de Log \%IT prev (esquerda), éimportante observar o alinhamento dospontos em relação à reta ajustada, bem como a distribuição dos pontos ao longo do intervalo de valores de atividades estudado. Já no gráfico de resíduos de regressão (direita), devese observar a distribuição dos resíduos, pois se espera que um modelo adequado aos dados observados tenha seus resíduos aleatoriamente dispersos em torno de zero. ${ }^{38}$

0 grau de significância desse modelo pode ser medido em termos do valor obtido para o teste $F$, comparando 0 mesmo ao valor de referência. Para um nível de confiança de $95 \%$, o valor de referência é $F_{[k, n-k-1]}=F_{[1,8]}=5,32 .{ }^{56}$ Como na equação 2 o valor encontrado foi bem maior, essemodelo apresentaum ótimo nível deconfiança. Q uanto ao grau de previsibilidade desta equação, o mesmo apresenta-se satiffatório, pois o $Q^{2}$ é de 0,701 e o SPRESS é bem próximo a zero.

O s demais modelos gerados com um descritor são apresentados a seguir:

$\log \%$ IT $=-0,01188( \pm 0,00619)$ RM $+3,13600( \pm 0,612723)$ $n=9 ; R^{2}=0,736 ; s=0,103 ; F=19,598 ; Q^{2}=0,565 ;$ SPRESS $=0,133$ $\log \%$ IT $=-0,00347( \pm 0,00202) M M+3,09006( \pm 0,657308)$ $n=9 ; R^{2}=0,692 ; s=0,112 ; F=15,719 ; Q^{2}=0,483 ;$ SPRESS $=0,145$ $\log \%$ IT $=-0,01899( \pm 0,01151)$ RM $3+2,33766( \pm 0,239807)$ $n=9 ; R^{2}=0,674 ; s=0,115 ; F=14,491 ; Q^{2}=0,387 ;$ SPRESS $=0,158$ 
$\log \%$ IT $=-0,00119( \pm 0,00078)$ VM $+3,15340( \pm 0,780879)$ $n=9 ; R^{2}=0,638 ; s=0,121 ; F=12,396 ; Q^{2}=0,391 ;$ SPRESS $=0,157$

A equação 3 demonstra estar de acordo com os pressupostos para aprovação na avaliação do grau de ajuste e significância, mas apresenta previsibilidade baixa. As equações 4, 5 e 6, apesar de apresentarem bons resultados para s e $F$, apresentam $R^{2}$ abaixo de 0,7 e previsibilidade ruim, pois os valores obtidos para validação cruzada estão abaixo do recomendado. $0 \mathrm{~s}$ gráficos da equação 6 são apresentados na figura 8, para que a qualidade do melhor (2) e do pior modelo (6) com um descritor possam ser comparadas visualmente.

Para modelos com dois descritores foram obtidas três equações:

Log\% IT $=-0,03166( \pm 0,02433)$ D z $-0,20513( \pm 0,05248)$ LogP $+3,00042( \pm 0,267172)$

$n=9 ; R^{2}=0,935 ; s=0,056 ; F=42,797 ; Q^{2}=0,841 ; S P R E S S=0,087$ (7) Log\% IT $=+0,00097( \pm 0,00112)$ AR 1-0,22786( $\pm 0,07945)$ LogP $+3,01130( \pm 0,344890)$

$n=9 ; R^{2}=0,900 ; s=0,069 ; F=27,044 ; Q^{2}=0,761 ;$ SPRESS $=0,106$ Log\% IT $=-0,00831( \pm 0,01002)$ RM 3 $-0,13397( \pm 0,08792)$ LogP $+2,79031( \pm 0,332463)$

$n=9 ; R^{2}=0,899 ; s=0,070 ; F=26,149 ; Q^{2}=0,761 ;$ SPRESS $=0,106$

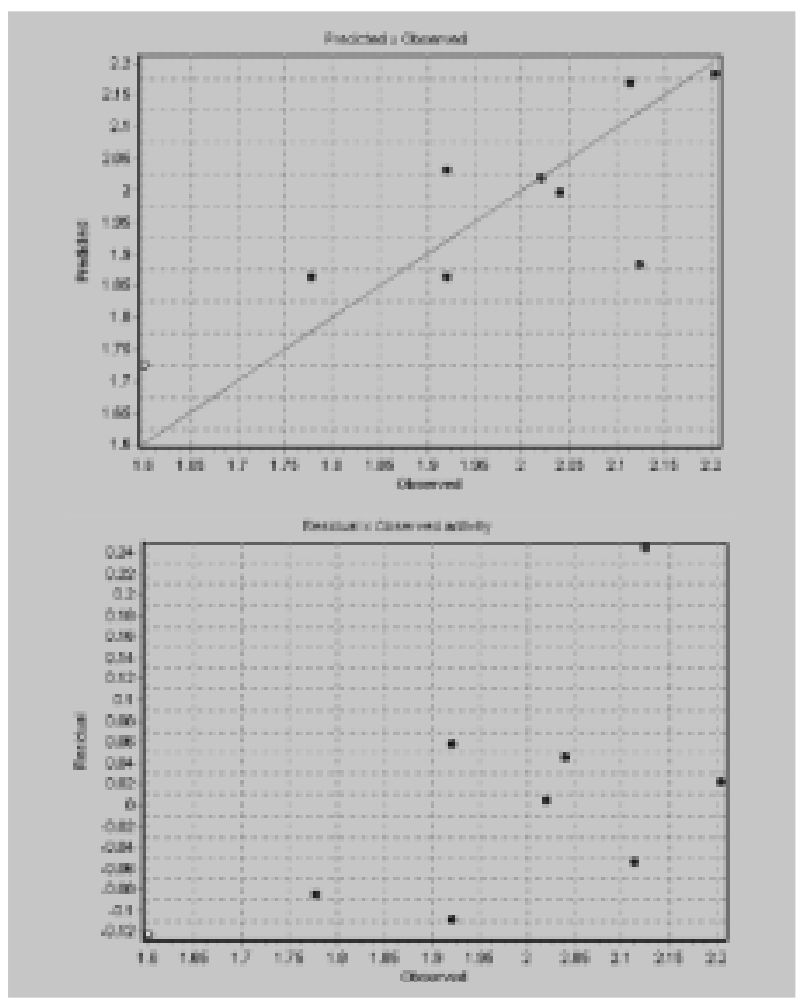

Figura 8. $\log \% \mathrm{IT} \mathrm{Dbs}_{\mathrm{bs}}$ em função de $\mathrm{Log} \% \mathrm{IT} \mathrm{T}_{\text {prev }}$ e resíduos de regressão em função de Log \%IT para a equação 6
As três equações foram ótimas quanto à avaliação do grau de ajuste, significância e previsibilidade. A equação 7 é a melhor entre todas, pois apresenta os melhores resultados para $\mathrm{R}^{2}, \mathrm{~s}, \mathrm{~F}, \mathrm{Q}^{2}$ e SPRESS (figura 9).

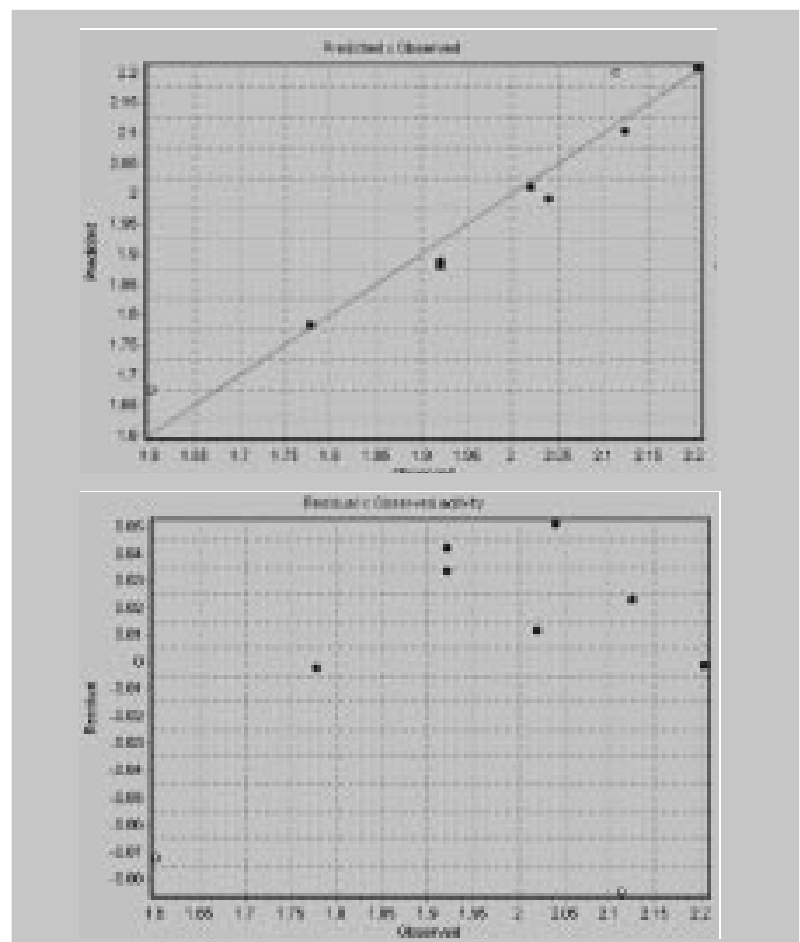

Figura 9. Log\%IT em função de Log \%ITPrev e resíduos de regressão em função de Log \%IT para a equação 7

A validação externa foi realizada conforme está descrito na metodologia, resultando assim em valores do quadrado do coeficiente de correlação da validação externa $\left(R_{\mathrm{VE}}^{2}\right)$ para os 8 modelos (tabela 3 ).

Tabela 3. Coeficientes de correlação obtidos pelo processo de validação externa $\left(R^{2}{ }_{v E}\right)$

\begin{tabular}{cc}
\hline Equações & $R^{2}{ }_{V E}$ \\
\hline 2 & 0,49 \\
3 & 0,44 \\
4 & 0,43 \\
5 & 0,97 \\
6 & 0,37 \\
7 & 0,50 \\
8 & 0,59 \\
9 & 0,67 \\
\hline
\end{tabular}

Pôde-se notar que todas as equações obtiveram valores abaixo do preconizado $\left(R_{V E}^{2}>0,7\right)$, exceto a equação 5. De forma interessante, é possível notar que 
a equação 7 , que apresentou os melhores valores para $R^{2}, s, F$ e $Q^{2}$, não apresentou o maior $R_{V E}^{2}$, e sim o terceiro melhor. Isto auxilia a comprovar a importância desta etapa, pois mostra que modelos de maior previsibilidade não serão necessariamente aqueles mais indicados para serem utilizados no planejamento da síntese de um novo derivado. ${ }^{33}$

A validação externa é uma etapa importante em estudos de QSAR, ${ }^{33}$ pois visa a enriquecer o modelo no sentido de comprovar a sua previsibilidade através da aplicação do mesmo a compostos da mesma classe e não utilizados na sua geração. Dessa forma, uma reprovação nesta etapa, em um caso como este, em que os outros modelos apresentaram-se eficazes, coloca as equações, principalmente 4 e 6 , em uma posição de menor enfoque, já que as duas também não obtiveram resultados tão satisfatórios na avaliação do grau de previsibilidade. Quanto à equação 5, apesar do altíssimo valor encontrado para a validação externa, esta não é possível de utilização devido a seus valores de $R^{2}$ e $Q^{2}$, indicando a existência de algum fator de variabilidade intrínseco e fora do controle.

Desta forma, após a avaliação dos graus de ajuste, significância e previsibilidade, os modelos $2,7,8$ e 9 demonstraram ser os melhores modelos encontrados. Apesar de seus valores para $R_{V E}^{2}$ terem ficado abaixo de 0,7 , este processo indica que os modelos em questão podem, teoricamente, prever corretamente o índice de inibição total da linhagem celular MCF7 de cerca de $49 \%$, 50\%, 59\% e 67\% dos novos compostos, cujas atividades possam ser previstas por estas equações. Esses melhores modelos foram então utilizados para a interpretação físico-química dos resultados.

De acordo com Montanari, Montanari e Gaudio, ${ }^{37}$ além de dizer se um certo parâmetro aumenta ou diminui a atividade biológica, a avaliação do coeficiente angular (constante pela qual é multiplicada a variável) fornece uma medida da influência do descritor frente à atividade biológica e sobre os demais descritores. Por exemplo, uma variável $X_{1}$ com um coeficiente de 0,3 apresenta menor influência sobre a atividade biológica do que uma $X_{2}$ com coeficiente de 1,0 . Desta forma, é necessário avaliar cada parâmetro e comparar seus coeficientes, para observar qual tem maior contribuição na atividade.

$\mathrm{Na}$ análise das equações selecionadas, observou-se uma grande contribuição da hidrofobicidade, e, em menor escala, de descritores eletrônicos e estéricos na atividade dos compostos estudados. Através das equaçōes 2, 7, 8 e 9, pode-se notar que um aumento do Log $\mathrm{P}$ leva a uma diminuição na atividade biológica em todas essas equações, pois o índice do coeficiente angular é negativo. Além do mais, a geração de modelos com dois descritores não causou um grande impacto na qualidade dos mesmos. Assim, a hidrofobicidade parece estar influenciando na inibição da linhagem MCF7 mais do que as outras propriedades.

A hidrofobicidade de um composto é um fator importante no planejamento de novos fármacos. Quando encontrada em um modelo, a mesma demonstra exercer forte influência em processos, tais como: absorção, ligação ao receptor e, se for o caso, transporte pela barreira hematoencefálica. Parâmetros hidrofóbicos também podem refletir uma combinação de propriedades estéricas e interações eletrostáticas dos compostos, dependentes principalmente de um distúrbio causado na estrutura da água. Essas características são correlacionadas com o tamanho e a área de superfície das moléculas, tendo uma relação direta com a água através da formação de pontes de hidrogênio. ${ }^{57}$

Considerando a contribuição negativa do descritor Log $\mathrm{P}$ nos modelos, isto nos leva a crer que os dibenzoilmetanos provavelmente tenham seu sítio de ação na superfície da célula, o que indicaria que, se estes compostos se difundirem facilmente pela bicamada lipídica da membrana celular, ou seja, forem facilmente absorvidos, a atividade diminuiria.

Com relação aos demais descritores presentes nos modelos (Dz, RM, RM3 e AR1), também é possível verificar uma contribuição negativa de propriedades estéricas e eletrônicas. O coeficiente angular destas variáveis em todas as seis equações selecionadas demonstra uma fraca contribuição quando comparado com o Log P. Por exemplo: a equação 7 é capaz de prever $94 \%$ da variabilidade. Todavia, ao se verificar a equação 2 , é possível ver que o Log P responde sozinho por $83 \%$ da variabilidade. Assim, apenas $11 \%$ é explicada por Dz. O mesmo ocorre na equação 9 , onde apenas $7 \%$ da variabilidade é explicada por RM3.

O momento de dipolo (D) é uma característica vetorial, e pode ser definido como a medida da magnitude da carga deslocada quando átomos de eletronegatividades diferentes são interligados. A direção do momento de dipolo de uma molécula é baseada nas eletronegatividades relativas dos átomos componentes e o valor é obtido pelo vetor resultante dos momentos de dipolo de cada eixo de ligação ( $x$, y e z) da molécula. ${ }^{57}$ A presença da variável $\mathrm{Dz}$ no modelo, cujo sinal de seu coeficiente angular é negativo, indica como a polarização na região da molécula, por onde passa o eixo z, influencia a atividade biológica dos compostos estudados. $\mathrm{O}$ sinal negativo pode indicar que esta região da molécula, quando da interação droga/receptor, estaria "tocando" um local de polarização de mesmo sinal (ou seja, polarização negativa com negativa ou positiva com 
positiva), o que desfavorece a formação do complexo. Sendo assim, novos derivados deverão, preferencialmente, apresentar valores baixos para $\mathrm{D} z$ ou de sinal negativo, o que favorecerá um aumento da atividade biológica. Porém, mesmo que este dado tenha permitido esta interpretação, a contribuição deste descritor para a atividade biológica é baixa.

Com relação aos modelos com os descritores estéricos RM, RM 3 e AR 1 , ficou caracterizado que 0 tamanho da molécula é inversamente proporcional à sua atividade. Esse fato é geralmente observado para compostos que se ligam a receptores pouco flexíveis e onde o sítio de ação é relativamente pequeno.

A refratividade molar ( $R M)$ é considerada uma característica mista, pois ao mesmo tempo em que ela é diretamenteproporcional ao tamanho da molécula, também éuma medida do quanto facilmenteum composto polarizase. $^{28}$ D esta forma, a equação 9 demonstra que a atividade desses compostos é favorecida na presença de substituintes pequenos em R3. Além disso, o sinal negativo do coeficiente angular também pode estar indicando, assim como ocorreu com D z, que a capacidade da molécula se polarizar é outro fator que desfavorece a atividade biológica. Estas informações, somadas às da equação 7 , sugerem que os dibenzoilmetanos possivelmente realizam interações eletrostáticas fracas com o sítio de interação no receptor, onde o grau de polarização da molécula é menor (por exemplo, forças de van der Waals).

A linhagem de células M CF 7 apresenta em seu núcleo receptores estrogênicos. As ações desses receptores podem ser mediadas com a ligação do composto diretamente no núcleo da célula, ou via segundos mensageiros liberados quando da ligação a receptores na membrana ou no citoplasma. Em um estudo de $Q S A R$, relacionando ${ }^{59}$ derivados de TMX com a afinidade de ligação ao receptor estrogênico citoplasmático presentes em células uterinas de ratas, demonstrou-se uma influência negativa de propriedades hidrofóbicas e estéricas na interação fármaco/receptor. D esta forma, em virtude da semelhança estrutural entre os tamoxifenos e alguns dos dibenzoilmetanos $\alpha$ substituídos (figura 9), especialmente 3, 6, 9, 11 e 12, nota-se um possível caminho a ser tomado com relação ao estudo do mecanismo de ação dos dibenzoilmetanos.

\section{CONCLUSÃO}

0 estudo deQ SAR, através da abordagem deH ansch, mostrou-se eficaz na avaliação da atividade antitumoral dos dibenzoilmetanos $\alpha$-substituídos. As equações geradas pelos modelos de regressão, quando bem avaliadas, podem ser usadas como ponto de partida para

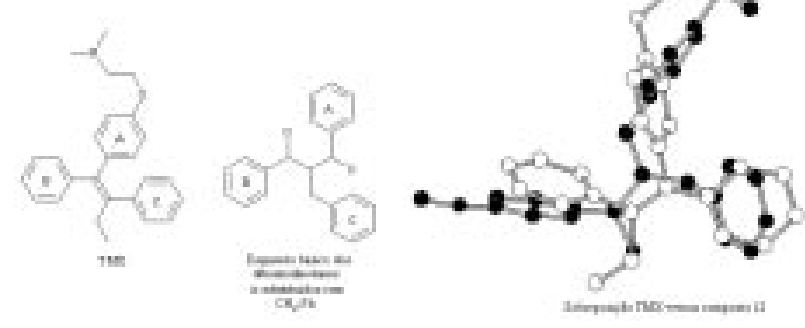

Figura 10. Comparação da estrutura do TMX e do esqueleto básico dos dibenzoilmetanos $\alpha$-substituídos com $\mathrm{CH}_{2}-\mathrm{Ph}$, e so breposição das estruturas tridimensionais do TMX (esferas brancas) e do composto 12 (esferas pretas). Sobreposição criada no HyperChem 7.1, utilizando as estruturas otimizadas pela metodologia descrita

a elucidação do mecanismo de ação e para o planejamento e síntese de análogos com perfil farmacológico otimizado.

O sresultadosobtidosnestetrabalho, apesar do pequeno número de compostos utilizados e dos baixos valores encontrados para $R_{V E}^{2}$, podem direcionar posteriores estudos sobre os dibenzoilmetanos e a atividadeantitumoral em linhagem decélulas M CF7. Através das equações, podese chegar à compreensão de quais propriedades são importantes para a atividade descrita. Essas informações, além de fornecerem importantes pistas quanto ao possível mecanismo de ação dosmesmos, auxiliam no planejamento de N.E.M . com potencial utilização como fármacos no tratamento do câncer da mama, ou como protótipos desses novos fármacos.

\section{AGRADECIMENTOS}

$O s$ autores agradecem à FUNDEP/U NIOESTE e ao CURSO DE FARMÁCIA DA UNIOESTE pelo apoio financeiro.

\section{REFERÊNCIAS}

1. Rang H P, D aleM M , Ritter JM , M oore, PK. Farmacologia. 5. th ed. Rio deJ aneiro: Elsevier; 2004. p. 789-809.

2. FariaSL, LemeLH S, O liveiraFilhoJA. Câncer demama. Rio deJaneiro: M ED SI; 1994.

3. Instituto N acional de Câncer; M inistério da Saúde. Programa $\mathrm{N}$ acional de $\mathrm{Controle} \mathrm{do} \mathrm{C}$ âncer do $\mathrm{C}$ olo do Ú tero edeM ama: VivaM ulher. [citado em 2006 Abr 24]. Disponível em: http://www.inca.gov.br/conteudo_ view. asp?id=140; 
4. Instituto Nacional de Câncer; Ministério da Saúde. Câncer de Mama. [citado em 2006 Abr 24] Disponível em: http:/ /www.inca.gov.br/conteudo_view.asp?id=336.

5. Instituto Nacional de Câncer. Câncer de mama. Rev Bras Cancerol. 2001;47:9-19.

6. Instituto Nacional de Câncer. Controle do câncer de mama: documento de consenso. Rev Bras Cancerol. 2004;50:77-90.

7. Callery PS, Gannett PM. Cancer and cancer chemotherapy. In: Williams DA, Lemke TL, editors. Foye's principles of medicinal chemistry. 5th ed. Baltimore (MD): Lippincott Williams \& Wilkins; 2002. p. 924-51;

8. Jordan VG. Selective estrogen receptor modulators. In: Williams DA, Lemke TL, editors. Foye's principles of medicinal chemistry. 5 th ed. Baltimore (MD): Lippincott Williams \& Wilkins; 2002. p. 1059-69.

9. Gilman AG, Hardman JG, Limbird LE. Quimioterapia das doenças neoplásicas. In: Goodman \& Gilman: as bases farmacológicas da terapêutica. 10th ed. Rio de Janeiro: McGraw-Hill; 2003. p. 1035-95.

10. Dados levantados com o programa SciFinder Scholar versão 2004.2, utilizando a palavra-chave Tamoxifen.

11. Haper MJ, Wapole AL. A new derivative of triphenylethylene: effect on implantation and mode of action in rats. J Reprod Fertil. 1967;13:101-19.

12. Marovac J. Investigación y desarrollo de nuevos medicamentos: de la molécula al fármaco. Rev Med Chile. 2001;129:99-106.

13. Korokolvas A. Planejamento de fármacos. Ciênc Cult. 1989; 41:528-37.

14. Chosi T, Horimoto S, Wang CY, Nagase H, Ichikawa M, Sugino E, et al. Synthesis of dibenzoylmethane derivatives and inhibition of mutagenicity in Salmonella Typhimurium. Chem Pharm Bull. 1992;40:1047-9.

15. Jackson KM, DeLeon M, Verret CR, Harris WB. Dibenzoylmethane induces cell cycle deregulation in human prostate cancer cells. Cancer Lett. 2002;178:161-5.

16. Wostyn K, Binnemans K, Clays K, Persons A. Molecular first hyperpolarizability data for lanthanate complexes containing the hemicyanine chromophore J Phys Chem B. 2001;105:5169-73.

17. Mabjiesh NJ, Willard MT, Harris WB, Sun H, Wang R, Zhong $\mathrm{H}$, et al. Dibenzoylmethane, a natural dietary compound, induces HIF-1a and increases expression of VEGF. Biochem Biophys Res Commun. 2003;303:279-86.

18. Nakano K, Nakayachi T, Yasumoto E, Morshed SR, Hashimoto K, Kikuchi H, et al. Induction of apoptosis by b-diketones in human tumor cells. Anticancer Res. 2004;24:711-7.

19. Blatt AF, Chávez D, Chai H, Graham JG, Cabieses F,
Farnsworth NR, et al. Cytotoxic flavonoids from the stem bark of Lonchocarpus aff. Fluvialis. Phytoter Res. 2002;16:320-5.

20. Dinkova-kostova AT, Talalay P. Relation of structure of curcumin analogs to their potencies as inducers of phase 2 detoxification enzymes. Carcinogenesis. 1999;20: 911-4.

21. Ragaswami S, Sastry BVR. Plant insecticides. VIII. Pods of Tephrosia lanceolata. Proc Indian Acad Sci Sec A. 1953;38A:257-9.

22. Narayanaswamy S, Rangaswami S, Seshada TR. Chemistry of pongamol. 2. J Chem Soc. 1954;1871-3.

23. Rao EV, Raju NR. Occurrence of (-)-isolonchocarpin in the roots of Tephrosia purpurea. Phytochemistry. 1979;18:1581-2

24. Rajani P, Sarna PN. Chemical examination of Tephrosia hamiltonii. J Indian Chem Soc. 1988;65:148-9.

25. Singh A, Ma D, Kaplan DL. Enzyme-mediated free radical polymerization of styrene. Biomacromolecules. 2000;1:592-6.

26. Biloti DN, Reis MM, Ferreira MMC, Pessive FBT. Photochemical behavior under UVA radiation of betacyclodextrin included Parsol 1789 with a chemometric approach. J Mol Struct. 1999;557:480-1.

27. Thomas G. Descoberta de fármacos por modelagem. In: Química medicinal: uma introdução. Rio de Janeiro: Guanabara Koogan; 2003. p. 23-72.

28. Tavares LC. QSAR: a abordagem de Hansch. Quím Nova. 2004; 27:631-9;

29. Sant'Anna CMR. Glossário de termos usados no planejamento de fármacos (recomendaçóes da IUPAC para 1997). Quím Nova. 2002;25:505-12;

30. Amaral AT, Montanari CA. Química medicinal: 25 anos de planejamento racional de fármacos. Quím Nova. 2002;25:39-44;

31. Csizmadia IG, Enriz RD. The role of computational medicinal chemistry in the drug discovery process. J Mol Struct. 2000;504:ix-x.

32. Nogueira MA, Marsaioli AJ, Pessine FBT, Biloti DN, Carvalho JE, Magalhães AF, et al, inventores; Obtenção de novos derivados de dibenzoilmetano que apresentam atividade antineoplásica e de aplicação potencial como filtro solar. Patente Brasileira. PI 6583-8. 2001.

33. Golbraikh A, Tropsha A. Beware of q2. J Mol Graphics Modell. 2002;20:269-76.

34. HyperChem Version 7.1. Gainesville (FL): Hypercube Inc; 2002.

35. Oliveira DB, Gaudio AC. BuildQSAR. Version 1.0.0 [monografia na Internet]. Vitória (ES): Universidade Federal do Espírito Santo; 2000. [citado em 2006 Abr. 24]. Disponível em: http://www.cce.ufes.br/anderson/htm/ buildqsar.htm.

36. OpenOffice. Version 1.1.5. Sun Mycrosystems; 2004. 
[citado em 2006 Abr. 24]. Disponível em: http:// www.openoffice.org.

37. Montanari MLC, Montanari CA, Gaudio C. Validação lateral em relaçōes quantitativas entre estrutura e atividade farmacológica, QSAR. Quím Nova. 2002; 25:231-40.

38. Gaudio AC, Zandonade E. Proposição, validação e análise dos modelos que correlacionam estrutura química e atividade biológica. Quím Nova. 2001;24:658-71.

39. Neves PJ, Costa JBN, Ndiyae PM, Carneiro JWM. TOP: um programa de cálculo de descritores topológicos para uso em correlaçōes entre estrutura e atividade. Quím Nova. 1998;21:709-13.

40. Eliezer JB, Rodrigues CR, Albuquerque MG, Sant'Anna CMR, Alencastro RB. Modelagem molecular: uma ferramenta para o planejamento racional de fármacos em química medicinal. Quím Nova. 1997;20:300-10.

41. Carvalho I, Pupo MT, Borges ADL, Lílian SCB. Introdução à modelagem molecular de fármacos no curso experimental de química farmacêutica. Quím Nova. 2003;26:428-38.

42. Ferreira MMC, Montanari CA, Gáudio AC. Seleção de variáveis em QSAR. Quim Nova. 2002;25:439-48.

43. Gilli P, Bertolasi V, Pretto L, Ferretti V, Gilli G. Covalent versus electrostatic nature of the strong hydrogen bond: discrimination among single, double, and asymmetric single-well hydrogen bonds by variable-temperature $\mathrm{X}$-ray crystallographic methods in beta-diketone enol RAHB systems. J Am Chem Soc. 2004;126:3845-55.

44. Costa P, Pilli R, Pinheiro S, Vasconcelos M. Substâncias carboniladas e derivados. Porto Alegre: Bookman; 2003.

45. Nogueira MA, Magalhães EG, Magalhães AF, Biloti DN, Laverde Jr A, Pessine FBT, et al. A novel sunscreen agent having antimelanoma activity. Fármaco. 2003; 58:1163-9.

46. Ferreira MMC. Multivariate QSAR. J Braz Chem Soc. 2002; 13:742-53.

47. Silva THA. Modelagem molecular. In: Andrei CC, Ferreira DT, Faccione M, Faria TJ, editors. Da química medicinal à química combinatória e a modelagem molecular: um curso prático. Barueri: Manole; 2003. p. 111-39.

48. Perekhodtsev GD. Computational chemistry introduction.
Review. Ab initio vs Semiempirical (CNDO, INDO, NDDO, MNDO, AM1, PM3) quantum chemical calculation. [citado em 2006 Abr 24]. Disponível em: http:/ /edu.mblogger.cn/jghx_1/posts/4061.aspx.

49. Karelson M, Lobanov VS, Katritzky AR. Quantumchemical descriptors in QSAR/QSPR studies. Chem Rev. 1996; 96:1027-43.

50. Melo EB, Bruni AT, Ferreira MMC. Validation of semiempirical methodology for geometry optimization of HIV1 inhibitors. In: Ferreira EI, editor. CIFARP 2005. Proceedings of the 5th International Congress of Pharmaceutical Sciences; 2005 Sep 25-28; Ribeirão Preto, São Paulo: Brasil; 2005. p. 265.

51. Lima Neto QA. Dibenzoilmetanos: modelagem molecular e avaliação inicial da correlação propriedades vs atividade anticâncer de mama [monografia]. Cascavel (PR): Universidade Estadual do Oeste do Paraná; 2005.

52. Lagier CM, Vila AJ, Olivieri AC. Shifting of the keto-enol equilibrium im 1,3-diphenyl-1,3-propanedione by methyl substitution. An AM1 study. J Mol Struct. 1994; 309:59-64

53. Chemical calculations. In: HyperChem ${ }^{\circledR}$ Release 7.0 for Windows: reference manual. Hypercube Inc; 2002. p. 287-470.

54. Alley MC, Scudiero DA, Monks A, Hursey ML, Czerwinski MJ, Fine DL et al. Feasibility of drug screening with panels of human tumor cell lines using a microculture tetrazolium assay. Anticancer Res. 1988; 48:589-601.

55. Costa Filho PA, Poppi RJ. Algoritmo genético em química. Quím Nova. 1999;22: 405-11.

56. Francisco W. Estatística básica. 2nd ed. Campinas: Editora Unicamp; 1993.

57. Van Waterbeemd H. Quatitative approaches to structure-activity relationships. In: Wermuth CC, editor. The practice of medicinal chemistry. San Diego: Academic Press; 1996. p. 367-89.

58. Costa MCA. Relações entre a estrutura química de neolignanas e a sua atividade antileishmaniose, envolvendo cálculos de solvatação [tese]. Campinas (SP): Universidade Estadual de Campinas; 1998.

59. Selassie CD, Garg R, Mekapati S. Mechanism-based QSAR approach to the study of the toxicity of endocrine active substances. Pure Appl Chem. 2003;75:2363-73. 